\title{
Automation of In-trench TEM Lamella Workflow Increasing Throughput for Lift- out
}

Marek Šikula ${ }^{1}$, Lukáš Hladík ${ }^{2}$, Jozef Oboňa ${ }^{2}$ and Rostislav Váňa ${ }^{1}$

${ }^{1}$ TESCAN Brno, s.r.o., Brno, Jihomoravsky kraj, Czech Republic, ${ }^{2}$ TESCAN ORSAY HOLDING, a.s., Brno, Jihomoravsky kraj, Czech Republic

Due to constant technological progress in semiconductor industry in recent years physical limits have been reached in manufacturing as well as in failure analysis. This puts enormous requirements on manufacturing processes and analytical methods. One of the integral tasks is the TEM lamella preparation, which is typically used across all departments from R\&D to Quality Control/Yield Ramp in the Production employing various approaches or geometries [1,2]. If less frequent non-repetitive device defects are left out, e.g. customer returns which are usually one of the kind and require mostly manual approach, one gets to the point where automation takes place to help to increase throughput and yield at any tool run by operators of various skill levels.

As one of the well-established vendors we would like to introduce our new solution for FIB-SEM systems which enables to manufacture TEM lamellae batch across the sample in an automatic manner up to socalled J-cut. In this abstract we present an optimized workflow for TEM lamella preparation on standard memory sample where multiple lamellae were prepared at different regions of interest. The sample was assessed from manufacturing and $R \& D$ point of view where any process changes have to be reviewed. These labs produce hundreds to thousands of lamellae a week and every time saving is well appreciated from economic point of view. In the paper our focus is on the initial part of TEM lamella preparation process prior to lift-out with minimum of user intervention.

In our tests we strove to minimize processing time so lamella size $10 \times 5 \times 1 \mathrm{um}(\mathrm{L} \times \mathrm{H}$ x D) was prepared while targeting the middle region to be thinned down to transparency later on. Overall process parameters were maximally optimized resulting to final processing time of 7 minutes for above mentioned geometry with the J-cut included.

Such optimized automated workflow offers considerable time and financial savings and allows to use FIBSEM systems capabilities to the maximum even for the novice users. Every second of the tool time can be usefully utilized using our new automated solutions. 


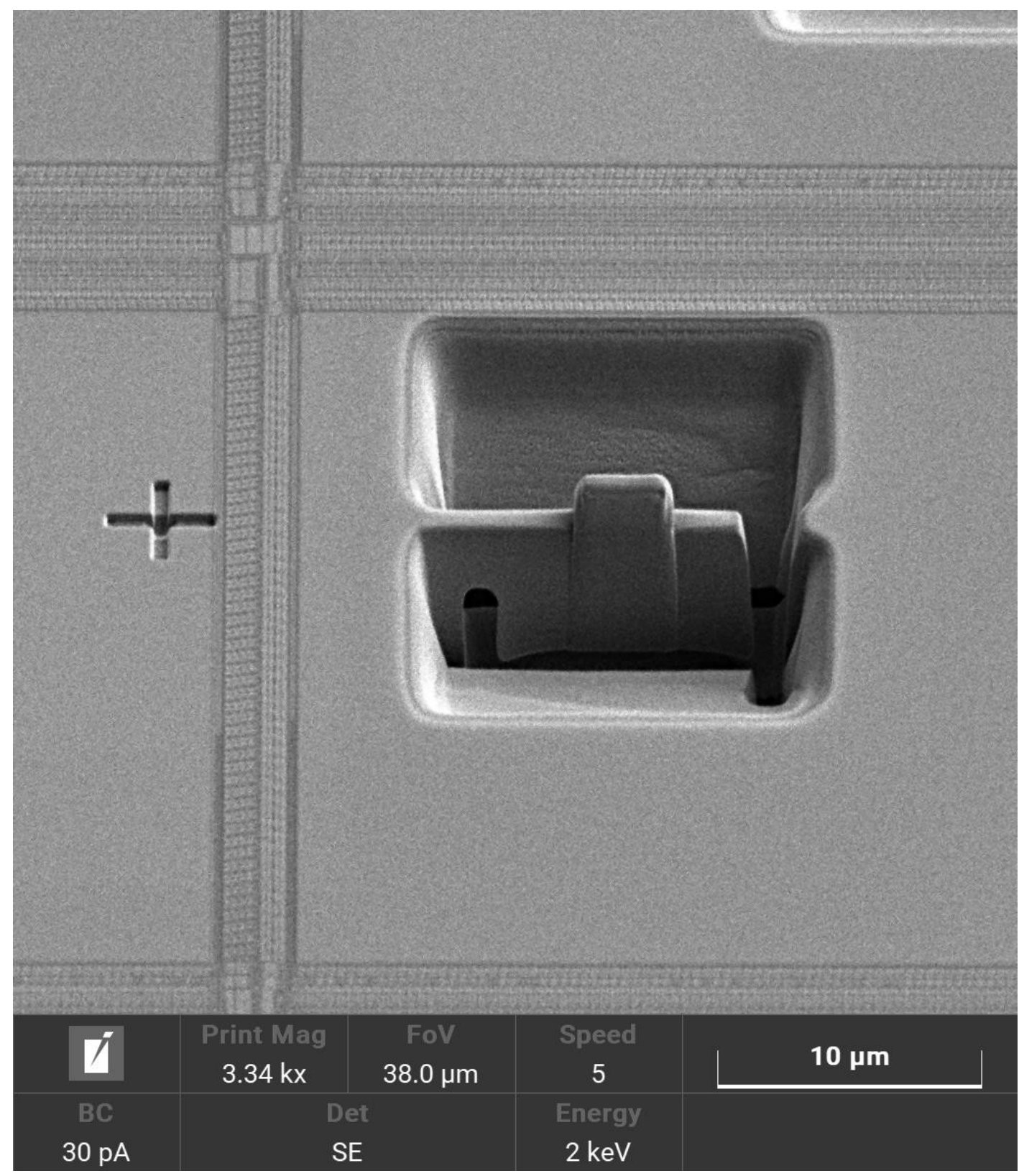

Figure 1. In-trench lamella prepared in an automatic manner within just 7 minutes including J-cut.

\section{References}

[1]: Z. Yuzhe et. al., "Advanced ex-situ TEM sample preparation for full metal process samples", 2017 IEEE $24^{\text {th }}$ International Symposium on the Physical and Failure Analysis of Integrated Circuits (2017), p. 1-3.

[2]: T. Hrnčíř et. al., "Variable Angle TEM Grid Holder for Advanced TEM Lamellae Preparation", ISTFA 2019: Conference Proceedings from the $45^{\text {th }}$ International Symposium for Testing and Failure Analysis (2019), p. 219-222. 